\author{
Military Technical College \\ Kobry El-Kobbah, \\ Cairo, Egypt.
}

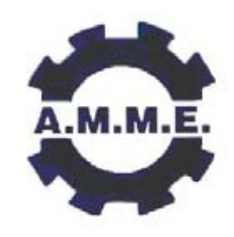

\section{$13^{\text {th }}$ International Conference on Applied Mechanics and Mechanical Engineering.}

\title{
LEAK DETECTION AND LOCALIZATION OF THE PIPELINE SYSTEMS
}

\author{
FOUAD ${ }^{(1)}$ M.M., AI-AWADI ${ }^{(2)}$ H., MOUSTAFA ${ }^{(3)}$ K.A.F. and NAWARA ${ }^{(4)}$ G.M.
}

\begin{abstract}
Leaks of pipeline contribute to the environmental pollution, promote corrosion and equipment failures and produce instabilities in the pipeline operation and control. The paper purpose is to develop a mathematical model that accounts for the behavior of pressure and flow-rate profiles in a pipeline systems and also involves simulation of pipeline conditions using advanced models which describe the fluid behavior in pipeline systems. The resulting advanced nonlinear mathematical model provides precise information on the actual flow and pressure in the whole pipeline system and describes the behavior of the underlying system. The proposed leak detection and localization method evaluates the mismatch between actual variables and those of healthy pipeline to detect leak. The proposed method appears attractive due to its simplicity. The traditional leak detection and localization methods are very expensive as they require the measurement of total pipeline flow and other variables at many points and are likely to produce unsatisfactory results due to ignoring the transient changes in the product variables.
\end{abstract}

\section{KEY WORDS}

Leak Detection, Leak Localization, Residual Time Average, Fault Diagnosis, Pipeline Systems, Kalman Filter, State Estimation.

\footnotetext{
(1) - (2) Lecturer, Industrial Engineering Department, Faculty of Engineering, Zagazig University.

(3) Professor Industrial Engineering Department, Faculty of Engineering, Zagazig University.

(4) Professor and chief Industrial Engineering Department, Faculty of Engineering, Zagazig University.
} 


\section{INTRODUCTION}

Pipeline Systems are the safest and most economical way to transport and distribute vast quantity of petroleum and its products from production fields and reservoirs to refineries and consumers. Pipeline system is defined as all portions of the physical facilities through which liquid or gas moves during transportation including pipe, valves, and other appurtenances attached to the pipe, such as compressor units, metering stations, regulator stations, delivery stations, holders and other fabricated assemblies [1].

There are several inherited problems regarding transportation of petroleum and its products in pipeline systems [2,3]. The supervision of pipelines is the subject of increased importance because of the increasing demands on stability and safety $[4,5,6]$. The unintentional release of fluid from pipelines is considered as leak. In addition to loss of products, leaks from pipelines pose potential hazards to the environment and concern for safety. Leaks contribute to the environmental pollution, promote corrosion and equipment failures and produce instabilities in the pipeline operation and control. Furthermore, pipeline leaks may have a severely damaging impact on the pipeline operating company's public reputation. There is a need, therefore, to develop an effective leak detection scheme in pipeline networks.

Pipeline leak may result, for example, from bad workmanship or from destructive causes such as those due to sudden changes of pressure, corrosive action, cracks, and defects in pipes or lack of maintenance $[7,8]$. Valve failure or malfunction, mechanical damage from third parties, digging close to pipelines and unauthorative cutting or drilling holes in pipelines and collection of the leakage to sell are also common causes of pipeline leaks.

It is apparent that most of the leak detection methods are very expensive to use because they require the measurement of total pipeline flow and other variables at many locations [9]. These methods are likely to produce unsatisfactory results due to ignoring the transient changes in the product variables such as temperature, pressure, gravity, density and viscosity. Moreover, most of the leak detection and localization methods discussed in the literature lack the capability of detecting small leaks. Although methods based on volume balance techniques can detect small leaks [10], their response time is long. Furthermore, the available leak detection methods are usually specialized to certain types of leaks.

It should be noted that many of the existing leak detection methods did not fully utilize the potential of real-time parameter identification techniques and the statistical decision making methodologies. In addition, the reported model-based detection methods have fallen short of providing reliable tests for the robustness of the detection and localization methods as well as their sensitivity to the modeling assumptions. [11].

The pipeline conditions during normal operation will be simulated and the state variables of the pipeline such as the pressure and flow rate will be computed in real time. This computation will be based on simulated measurements of pipeline variables such as flow rate and pressure collected at few selected locations along the pipeline. The objective of the simulation is to study the performance of the considered pipeline under the effect of different leak conditions. The developed mathematical model is solved numerically under the given boundary conditions. The response of the pressure 
and flow rate at different nodes along the pipeline are obtained. The pressure and flow rate distributions at selected time instants also are obtained.

The circle graph of Fig. 1 indicates the importance of pipeline system compared with other tools of liquids and gas transportation. Pipelines are considered, moreover, the safest and most economic way of transporting gas or liquids over long distances. Because of the volume that must be transported, pipelines are the only feasible economical method for moving the enormous quantities of gas or liquids. In addition to their efficiency, pipelines also have important environmental and safety benefits [12]. Compared to other inland transport modes, pipelines do not crowd highways and rivers and they produce negligible air pollution. Pipelines also have a lower spill rate per barrel of oil transported than competing modes of transportation.

\section{CAUSES OF PIPELINE SYSTEM LEAK}

A pipeline system consists of many components such as, pipes (main pipe and connection pipes), valves, pumps, pump stations and compressors. Malfunction of any of the pipeline elements may cause a leak or even multi leaks. Fig. 2 shows the causes of PLS element malfunction by using fish-bone (cause-and-effect) tool. There are many types of faults that can cause leaks in pipeline system $[4,13,14]$.

\section{PROPOSED LEAK DIAGNOSIS SYSTEM}

The proposed diagnosis system involves developing mathematical model that accounts for the transient behavior of pressure and flow rate profiles in the pipeline systems. This involves computational pipeline monitoring and simulation of pipeline conditions using advanced models that describe the fluid behavior in pipeline systems. The conservation laws of momentum, energy and mass are typically applied in the modeling process The resulting advanced nonlinear mathematical model provides precise information on the actual flow and pressure in the whole pipeline system.

\section{STRUCTURE OF THE PROPOSED SYSTEM}

The general structure of the leak detection and localization method is shown in Fig. 3 . It is based on a priori knowledge and experience of the real pipeline system operational conditions and is composed of two modeling blocks; model of the normal pipeline pressure and flow rate profiles and model of faulty pipeline pressure and flow rate.

The pipeline conditions during normal operation is simulated and the state variables of the pipeline are computed in real time. This computation are based on simulated measurements of pipeline variables collected at few selected locations along the pipeline.

\section{LEAK FUNCTION}

Theoretically, leak function can represent the relationship between leak amount, volume, mass out from a hole, or leak rate, as a dependent variable with time, pipe 
diameter, leak distance, pipe elevation, pipe length, valves/pump performance, ambient temperature, surrounding temperature, hole geometry, commodity temperature, ...... etc, either all or some of them. To this end, the following leak function is introduced.

$Q_{L}(z, t)=\int_{0}^{z} \sum_{i=1}^{n} Q_{i}(t) \delta\left(z-z_{i}\right)$

It is assumed that the pipeline leaks at locations $z_{1}, z_{2}, \ldots . ., z_{n}$, where, $z_{1} \leq z_{2} \ldots . . \leq z_{n_{L}}$. Here, $n$ represents the number of leaks along the pipe line and $\delta($.$) is the Dirac delta$ function. The leak rate at location $z_{i}$ is given by $Q_{i}(t)$. One can easily show, using Equation (1), that the leak function at a location $z$, where $z_{i} \leq z \leq z_{i+1}$, can be evaluated as

$Q_{L}(z, t)=Q_{i}(t)$

The above defined leak function is used in the following section to characterize the leak amount in the mathematical model of the pipeline system.

\section{PIPELINE MATHEMATICAL MODEL}

One-dimensional isothermal flow of a fluid in a horizontal pipeline with constant area $A$ and length $L$ will be considered.. The friction coefficient $f$ is assumed constant along the pipeline and the head loss due to friction is calculated according to the DarcyWeisbach formula. Most of the previous work dealing with leak diagnosis in pipelines considered only a single leak that is located at a certain position along the pipeline [8, $15,16,17]$. In the present paper, the general case of multi leaks occurring at different locations along the pipeline is introduced. The mathematical model given in references $[8,18,10]$ that describes the flow in a pipeline with single leak is modified in this paper. This mathematical model is summarized below for the convenience of the reader. Consider a control volume of length $\Delta z$ and applying the mass and momentum balance equations, the following first order partial differential equations representing the dynamics of the flow in the considered pipeline are obtained.

$\frac{\partial p}{\partial t}+\frac{a^{2}}{A} \frac{\partial q}{\partial z}+\frac{a^{2}}{A \Delta z} q_{L}=0$

$\frac{\partial q}{\partial t}+A \frac{\partial p}{\partial z}-\frac{a^{2}}{A \Delta z} \frac{q}{p} q_{L}=-\frac{f}{2 D} \frac{a^{2}}{A} \frac{q|q|}{p}$

where $D$ is the pipe diameter and $a$ is the wave speed of sound in the pipeline system by $\mathrm{m} / \mathrm{s}$.

\section{PIPELINE MATHEMATICAL MODEL WITH MULTI LEAKS}

Using the leak function as defined by Equation (1) in Equations (3) \& (4), the mathematical model describing the flow in a pipeline with multi leaks can be written as: 
$\frac{\partial p}{\partial t}+\frac{a^{2}}{A} \frac{\partial q}{\partial z}+\frac{a^{2}}{A \Delta z} Q_{L}(z, t)=0$

$\frac{\partial q}{\partial t}+A \frac{\partial p}{\partial z}-\frac{a^{2}}{A \Delta z} \frac{q}{p} Q_{L}(z, t)=-\frac{f}{2 D} \frac{a^{2}}{A} \frac{q|q|}{p}$

The last terms on the right hand side of Equations (5) and (6) represent multi leaks at different locations along the pipeline. This describes the leak in the pipeline in a more rigorous way where the leak rate as well as the leak location are explicitly included in the model. The shortcoming of the formulation of Equations (1) and (2) in the sense that only a single leak is considered and the leak location is known, is obviously eliminated by the introduction of the leak function in Equations (5) and (6). These equations are solved numerically using MatLab. Toward that end, let the pipeline be divided into equal segments using $N$ nodes as indicated in Fig. 4 . The derivatives in the equations will be approximated using the alternative Crank-Nicolson scheme with backward timecentered space $[8,19,20,21]$. Assembling the resulting discretized equations of the $\mathrm{N}$ nodes, the following state space representation is obtained.

$$
A X(t)=B X(t-1)+C(X(t-1))+E(X(t-1)) \bar{Q}_{L}
$$

where the state vector $X(t)$ is defined in terms of the nodal pressures $p_{i}(t)$ and flow rates $q_{i}(t), i \in\{1,2, \ldots, N\}$ as:

$$
\begin{gathered}
X(t)=\left[X_{p}^{T}(t), X_{q}^{T}(t)\right]^{T} \quad \text { where : } \\
X_{p}(t)=\left[p_{1}(t), p_{2}(t), \ldots \ldots, p_{N}(t)\right]^{T} \text { and } X_{q}(t)=\left[q_{1}(t), q_{2}(t), \ldots \ldots, q_{N}(t)\right]^{T}
\end{gathered}
$$

The leak input vector for $\mathrm{n}$ leaks is defined as $\bar{Q}_{L}=\left[\begin{array}{llll}Q_{1} & Q_{2} & \cdots & Q_{n}\end{array}\right]^{T}$,Here, the superscript $\mathrm{T}$ denotes the transpose operation of a matrix or a vector. The system matrices of Equation (7) are defined as:

$$
\begin{aligned}
& A=\left[\begin{array}{cc}
I_{N \times V} & 0.5 \alpha P \\
0.5 \beta P & I_{N \times N}
\end{array}\right] \quad B=\left[\begin{array}{cc}
I_{N \times N} & -0.5 \alpha P \\
-0.5 \beta P & I_{N \times N}
\end{array}\right] \quad C=-\frac{f}{2 D} \frac{a^{2}}{A} \Delta t\left[\begin{array}{c}
0_{N \times 1} \\
\bar{C}_{N \times 1}
\end{array}\right] \\
& \overline{C_{N \times 1}}=\left[\begin{array}{c}
q_{1}\left|q_{1}\right| / p_{1} \\
q_{2}\left|q_{2}\right| / p_{2} \\
\cdot \\
\cdot \\
q_{N}\left|q_{N}\right| / p_{N}
\end{array}\right] \quad E=\left[\begin{array}{c}
E_{p} \\
E_{q}
\end{array}\right]
\end{aligned}
$$

Assuming that the $k^{\text {th }}$ leak occurs at the $n_{k}$ node, the elements of the $E_{p}(N x n)$ matrix are given by:

$$
\begin{array}{ll}
E_{p}\left(n_{k}, k\right)=-2 \alpha \frac{q_{n_{k}}}{p_{n_{k}}}, \quad k \in\{1,2, \ldots, n\} & \\
E_{p}(i, j)=0, & \text { Otherwise }
\end{array}
$$


Similarly, the elements of the $E_{q}(N x n)$ matrix are given by:

$$
\begin{aligned}
& E_{q}\left(n_{k}, k\right)=-2 \alpha \frac{q_{n_{k}}}{p_{n_{k}}}, \quad k \in\{1,2, \ldots, n\} \\
& E_{q}(i, j)=0, \text { Otherwise }
\end{aligned}
$$

The parameters $\alpha$ and $\beta$ in the above equations are given by: $\alpha=\frac{a^{2} \Delta t}{2 A \Delta z} \quad \beta=\frac{A \Delta t}{2 \Delta z}$ Where $\Delta t$ and $\Delta z$ denote, respectively, to the time step and the segment length of the discretization scheme.

\section{MODEL SIMULATION AND LEAK DETECTION}

The mathematical model developed in previous section is simulated in this section by using the MatLab package. The objective of the simulation is to study the performance of the considered pipeline under the effect of different leak conditions. The response of the pressure and flow rate at different nodes along the pipeline and the pressure and flow rate distribution at selected time instants are obtained. The state space model of Equation (7), that represents the mathematical model of the underlying pipeline system is solved by using MatLab with the pipeline data given in the example presented in [8]. The pipeline discussed in this example is assumed to carry crude oil and has the following specifications:

$$
\begin{array}{ll}
\text { pipeline length } L=90 \mathrm{~km} . & \text { Pipe diameter } D=1.1284 \mathrm{~m} \\
\text { Coefficient of friction } f=0.003 & \text { Speed of sound } a=300 \mathrm{~m} / \mathrm{s} .
\end{array}
$$

The boundary conditions are assumed such that the pressure at the inlet and outlet of the pipeline are, respectively, 3.5 bar and 1 bar. The initial condition is taken as $q_{1}(0)=24 \mathrm{~kg} / \mathrm{s}$. The discretization scheme is carried out with a time step $0.2 \mathrm{~s}$ and number of nodes 101 (100 segments with the length of each segment $\Delta z=900 \mathrm{~m}$ ). The simulation is first carried out for a healthy pipeline without any leak. Next, the case of two leaks occurring at nodes number 5 and 15 (which corresponds to locations $z_{1}=18 \mathrm{~km}$ and $\left.z_{2}=63 \mathrm{~km}\right)$ is simulated. The results of the simulation are shown in Fig. 6 through Fig. 16.

All simulation experiments were run for a total of 400 time instants with time step $d t=0.2 s$. The number of measurements taken for the pressure and flow rate along the pipeline was three for each. The leak detection scheme to be discussed would be based only on these six measurements rather than on all the 202 measurements of pressure and flow rate at the pipeline nodes. This obviously have the advantage of tremendously reducing the number of measurements needed in an actual pipeline for the purpose of leak diagnosis. This naturally leads to the reduction of the instrumentation needed as well as saving time and effort as a result of dealing with less number of data.

Three cases will be discussed. Case 1 will address the simulation issue of a healthy pipeline without any leak. The simulation of a faulty pipeline with a single leak will be discussed in Case 2. The leak detection technique by using Kalman filter, proposed in this work, will be described by considering a pipeline with a single leak in Case 3. 


\section{Case 1: Simulation of a Pipeline without leak}

In this case, a healthy pipeline without any leak will be simulated. The purpose is to investigate the behavior of the pipeline variables. Both the pressure distribution along the pipeline as well as the flow rate at the pipeline nodes will be calculated at different time instants. The results of the simulation are shown in Figures. 5 to 8 . The pressure and the flow rate profiles at the final time (400s) are shown, respectively, in Fig. 5 and Fig. 6. It can be seen that the pressure and flow rate distribution is smooth without any jumps or drops along the pipeline. On the other hand, the pressure and flow rate time responses at the middle node (node number 51) of the pipeline are shown, respectively, in Fig. 7 and Fig. 8. Again smooth response for both the pressure and flow rate can be observed.

\section{Case 2 - Simulation of a Pipeline with a single leak}

In this case, a single leak is introduced at the middle node of the pipeline; namely, at node 51 at time 200s. The corresponding mathematical model with the appropriate boundary and initial conditions is simulated. Both the pressure distribution along the pipeline and the flow rate at the pipeline nodes are numerically calculated at different time instants. The results of the simulation are shown in Figures 9 to 14. The pressure distribution along the pipeline $50 \mathrm{~s}$ after the onset of a leak is shown in Fig. 9. One can easily observe that there are some irregularities around the leaky node as a result of leak occurrence. The pressure distribution just before and just after the onset of a leak is also calculated and the results are shown in Fig. 10. It can be seen that the pressure after the onset of the leak is less than that before the onset of that leak in a region close to the leaky node.

The flow-rate distribution along the pipeline after the onset of leak by ten seconds is shown in Fig. 11. Again some irregularities can be seen in a region close to the leak location. The flow-rate distribution just and before the onset of leak is also shown in Fig. 12. It can be observed that there is a sudden increase in the flow rate just before the leak location followed by a sharp decrease after the leak location.

On the other hand, the pressure and flow-rate time responses at the middle node (node number 51) of the pipeline are shown, respectively, in Fig. 13 and Fig. 14. In Fig. 13, a noticeable drop in the pressure can be observed at the leak location just after the onset of leak at time 200s. Fig. 14 shows the upstream and the downstream flow rate response at the leak location. As shown in the figure, a sudden increase in the flow rate occurs upstream as compared to a decrease downstream the leaky node. Also shown in this figure is the flow rate response under no leak conditions

\section{Case 3 - Single leak detection of a Pipeline using Kalman filter}

In this case, a simulation experiment is run with a single leak of $10 \mathrm{~kg} / \mathrm{s}$ introduced at the middle node of the pipeline described above. The leak is assumed to suddenly occur at the time instant of 201s. Three measurements of each of the pressure and flow rate at different locations of the pipeline are taken as described above. Using these six measured values of the ipeline variables, the model state vector is constructed using Kalman filter $[22,23,24]$, assuming no leak. The average residuals of the pressure and flow rate along the pipeline are computed as functions of time [25]. The computed average residuals of flow-rate are plotted in Fig. 15 for the whole time period of the 
experiment of 400s. The computed average residuals of the pressure are similarly plotted in Fig. 15 and Fig.16. The figures clearly show that the absolute value of both the pressure and flow rate residual average sharply increase after the onset of leak. This characteristic can be used as an indication of the occurrence of a leak starting at the time when the residuals start to rise in magnitude.

The leak can thus be detected by calculating the average residual of both the pressure and flow rate of the pipeline. The result is shown in Table 2 which indicates clearly that the computed average residuals are considerably larger for the case of leak than that of no leak. Hence, by taking only six measurements along the pipeline, and by comparing the average residuals of the actual pipeline to those of the healthy one, which are computed online during normal operation, leak occurrence can be detected.

\section{PIPELINE LEAK DETECTION}

Leak localization processes is implemented by simulation experiment. It avoids the disadvantage of the traditional methods such as fiber optic in-line distributed sensors and fiber bending distributed sensors [26, 27, 28] and fluid transient model where these methods require use of many sensors along the pipeline [29]. The location of the leak can be determined by conducting a simulation experiment in which the leak is assumed to occur at a certain node. Then calculate the pressure and flow rate residual time average for this assumed leak location along the pipeline. This experiment is repeated for all the nodes along the pipeline starting from the input side. It is obvious that, when the assumed leak location coincides with the real one, the actual system state variables are exactly equal to the filtered ones. As a result, the residual time average for both the pressure and flow rate should be zero in this case. It should be noted that only few measurements (six in our simulation) are used in this localization scheme. This is a major advantage as compared to other schemes where all the pressure and flow rate measurement at all of the pipeline nodes are needed.

\section{Leak Localization Via Residual Scanning}

Leak localization is carried out by scanning the pipeline from the inlet node to the outlet one. At each node the scanning scheme calculates the average residual of the pressure and flow rate assuming that the leak occurs at this node. The scanning scheme is trying to find the node at which the minimum residuals are obtained. This ensures that this node is the one at which the actual leak occurs.

Two examples are simulated in this section. In Example 1, a leak rate of $10 \mathrm{~kg} / \mathrm{s}$ is considered, while a relatively small leak of about $5 \mathrm{~kg} / \mathrm{s}$ is considered in Example 2.

\section{Simulation Example 1}

A leak of flow rate $10 \mathrm{~kg} / \mathrm{s}$ is introduced at the middle node (node number 51 ) of the pipeline. This leak represents about $14 \%$ of the healthy pipeline flow rate at steady state. The residual time average for the pressure and flow rate along the pipeline is generated according to the proposed scheme. Fig. 17 and Fig. 18 show the results of the proposed leak localization scheme. In these figures negative and positive integers on the $\mathrm{x}$-axis represent, respectively, nodes located before and after the middle of the $\mathrm{PL}$. Therefore, 0 on the $\mathrm{x}$-axis indicates the location of the actual leak which is 
introduced at node number 51, as expected, the pressure and flow-rate residual average drop to zero as the correct leak location since the assumed leaky node coincides with the actual one. At any other location, however, it is expected to have larger residual because the assumed leak position does coincides with the correct one. Numerical error and noise effects could be the causes of the spikes shown a little after and before the correct location of leak in Fig. 17 and Fig. 18.

\section{Simulation Example 2}

The same simulation experiment is repeated but for a relatively small leak rate to test the effectiveness of the localization scheme in localizing small leaks. A leak of flow rate $5 \mathrm{~kg} / \mathrm{s}$ is introduced at the middle node (node number 51) of the pipeline. This leak represents about $7 \%$ of the healthy pipeline flow rate at steady state. The residual time average for the pressure and flow rate along the pipe line is generated according to the proposed scheme. Fig. 19 and Fig. 20 show the results of the simulation of this example. It is obvious that, even for this small leak, the leak location can still correctly be identified at the middle of the pipeline. The minimum average in both the pressure and flow-rate are still obtained at the correct leak location. However, the noise and numerical error effects become more dominant since the noise to signal ration is almost doubled.

\section{Leak Localization Via Flow rate and Pressure Responses}

An alternative method that can be used to double check the leak location as found by the residual method is discussed herby. This method is based on the characteristics of the flow rate and pressure responses of the pipeline when leak occurs. The flow rate response is recorded at two consecutive nodes; one just before the leak location and the second just after the leak location as shown in Fig. 21. The nominal flow rate response in the case of no leak is also shown as solid lines in the figures. As expected, the flow rate at the node before the leak location is higher than the corresponding value for the healthy pipeline. On the other hand, the flow rate at the node located after the leak location is lower than those of the healthy pipeline. This is intuitive since the occurrence of leak causes the pressure at the leak location to suddenly drop causing a higher flow rate upstream the leak and lower flow rate down stream the leak. This result coincides with the results illustrated by [30].

The pressure response at the leak location can also be used for checking the leak location. Fig. 22 shows the pressure response of the leaky pipeline as measured at the leaky node. As it is clear from this figure, the pattern obtained for the pressure response is similar to that expected at leak location.

\section{Estimation of amount of leak}

The results depicted in Figures from Fig. 18 to Fig. 21 prove that the pattern of flow rate residual average is independent of the amount of leak. Therefore, leak localization can be carried out once leak is detected without the need of knowing the amount of leak flow rate. The leak flow rate can, however, be estimated after detection and localization has been done. The leak flow rate can be estimated by calculating the difference between the flow rates of a healthy pipeline and the leaky one at any measuring station downstream the leaky node localized. 
Figure 23 shows the simulation results for the flow rate response, in case of leak rate of $10 \mathrm{~kg} / \mathrm{s}$, at the two consecutive nodes just downstream the leaky node. As expected, because of the leakage loss, the flow-rate downstream is less than of the normal flowrate by the amount of leak. The amount of leak flow rate can correctly be estimated from this figure as about $10 \mathrm{~kg} / \mathrm{s}$. This estimate is obtained by subtracting the steady state flow rates of the leaky pipeline from that of the healthy pipeline at any of the downstream nodes. It should be noted that the estimation of the amount of leak is essential to the pipeline company management to estimate the cost of lost material.

\section{FEATURES OF THE PROPOSED LEAK DIAGNOSIS SYSTEM}

Many of the reported leak detection methods may be affected by operational factors that may contribute to a deterioration of performance. Some of these operational factors that require further research are multiphase flow, compressible flow, slack-line effects, pre-existing leaks, variations in temperature, pressure and flow conditions, connected production areas, false alarms, pipeline abnormalities, fluid surge and instrumentation errors especially for pipelines with large. In the light of these drawbacks, the present paper aims at developing an effective leak diagnosis scheme capable of detecting leaks in pipeline systems while avoiding some of these drawbacks. Our proposed scheme possesses, as compared to previous work, the following features and advantages:

1. Real-time process models that account for the transient behavior of the pressure and flow rate profiles of the pipe line network as well as multi leak conditions.

2. The developed model is capable of detecting leaks in the pipeline network. It also can produce accurate estimates of the actual flow rates and pressures at any location of the whole pipeline system. These features are particularly attractive to the application of modern control theory methodologies, and will certainly save time and cost of conducting a large number of field measurements that are required by other conventional methods.

3. The presence of noise is inevitable in any practical system dealing with real measurements and it could seriously degrade the performance of the detection schemes that are based on these noisy measurements. The proposed approach will tackle this problem by developing better noise filtering techniques that reduce the detrimental effects of the noise on the detection results.

4. The diagnosis system can assist in pipeline daily operation by visualizing what is happening in a pipeline even with few instrumentation. The profile plot produced by the scheme approximates adding additional instrumentation on the pipeline and allows controllers to visualize concepts that are otherwise difficult to grasp e.g. watching a pressure wave moving down the pipeline.

5. Data storage and playback capability of the scheme may help in training as well as understanding how undesirable operation conditions developed.

A comparison between the proposed leak diagnosis method and some other conventional methods in Table 1 in which four criteria are used for comparison. The table clearly shows that the proposed method is the best with reference to all of the considered criteria. 


\section{CONCLUSION}

The proposed method appears attractive due to its simplicity. The traditional leak detection and localization methods are very expensive as they require the measurement of total pipeline flow and other variables at many points and are likely to produce unsatisfactory results due to ignoring the transient changes in the product variables. Moreover, most of these techniques lack the capability of detecting small leaks. The pipeline conditions during normal operation was simulated and the state variables were computed in real time based on simulated measurements collected at few selected locations along the pipeline and used the Kalman filter state estimation for detection of leaks. The Leak localization was carried out by scanning the pipeline from the inlet node to the outlet one. At each node the scanning scheme calculates the average residual of the pressure and flow rate assuming that the leak occurs at this node. The scanning scheme is trying to find the node at which the minimum residuals are obtained. This ensures that this node is the one at which the actual leak occurs. After detecting the leaks, the corrective actions should be taken by the operators or control engineers.

\section{REFERENCES}

[1] American Petroleum Institute (API), "Computational Pipeline Monitoring", API Publication 1130, 17 p, 1995.

[2] Shields, D. N., Ashton, S.A., and Dalys, S., "Design of a Nonlinear Observers for Detecting Faults in Hydraulic Sub-Sea Pipelines" Control Engineering Practice, Vol. 9, pp. 297-311, 2001.

[3] Shields, D. N., Ashton, S.A., and Dalys, S., "Robust Fault Detection Observers for Nonlinear Polynomial Systems", International Journal of Systems Science, Vol. 32, No. 6, pp. 723-737, 2001.

[4] Isermann, R., "Process Fault Detection Based on Monitoring and Estimation Methods-A Survey”, Automatica, Vol. 20 ,N. 4, pp 387-404, 1984.

[5] Isermann, R., and Freyermuth, B., " Process Fault Diagnosis Based on Process Model Knowledge- Part II: Case Study Experiments", Journal of Dynamic Systems, Measurement, and Control, Vol. 113, pp. 627-633, December 1991.

[6] Billmann, L. and Isermann, R., "Leak detection methods for Pipelines", Automatica, Vol. 23, Issue 3, pp. 381-385. 1987.

[7] Verde, C., "Accommodation of Multi-Leak Location in a Pipeline," Control Engineering Practice, Vol. 13, pp. 1071-1078, 2005.

[8] Shabaik, H.E., Khulif, Y.A. and Hussaini, I., " A Non-Linear Multiple State Estimation Scheme for Pipeline Leak Detection and Isolation ", Journal of Systems and Control Engineering, Vol. 216, No. 6, pp. 497-512, 2002.

[9] Yoon, M. S., Mensik, M., and Luk, W. Y., "Canadian Pipeline Installs LeakDetection System", Oil \&Gas Journal, pp77-85, May 30, 1988.

[10] Liou, J. C. P., and Tian, J., "Leak Detection -Transient Flow simulation Approaches", Journal of Energy Resources Technology, Vol. 117, pp. 243-248, September 1995.

[11] Stecki, J. S. and Davis, D. C. "Fluid Transmission Lines - Distributed Parameters Models Part 1: A Review Of The State Of The Art", Proceeding of the Institution of Mechanical Engineers, Vol. 200, No. 4, pp. 215-229, 1986. 
[12] Hunt, G.L. " Environmental Handbook, Environmental Regulations for the Oil \& Gas Exploration and Production Industry", Utah Division Of Oil, Gas \& Mining , January 1996.

[13] Kutokov, S.E., Bakhtizin, R.N. and Shammazov, A.M., "Gas Congestion Influence on Pipeline Systems Curve", Intellectual Service for Oil \& Gas Industry: Analysis, Solutions, Perspectives, Vol. 2, pp. 75-85, 2003.

[14] Leeds, J. M. and Grapiglia, J., "The DC Voltage-Gradient Method for Accurate Delineation of Coating Defects on Buried Pipelines", Corrosion Prevention and Control, Vol.42, No. 4, pp.77-86, 1995.

[15] Ashton, S.A., and Shields, D. N., " Fault Detection Observer for a Class of Nonlinear System", New directions in Nonlinear Observer Design, London, UK, Vol. 244, 1999.

[16] Ashton, S.A., Shields, D. N., and Dalys, S., "Fault Detection in Pipelines using Nonlinear Observers", IEEE Conference Publication, No. 455, pp. 135-140, 1998.

[17] Ashton, S.A., Shields, D. N., and Dalys, S., "Application of a Fault Detection Method for Pipeline Systems", Systems Sciences , Vol. 23, No. 2; pp. 97-109, 1997.

[18] Shields, D. N , "Models, residual design and limits to fault detection for a complex multi-tank hydraulic control system", International Journal of Control, Vol. 76, No.8, pp.781 - 793, 1997

[19] Tiana, Z.F., and Geb, Y.B., "A Fourth-Order Compact ADI Method For Solving Two-Dimensional Unsteady Convection-Diffusion Problems", Journal of Computational and Applied Mathematics Vol. 198, pp. 268-286, 2007.

[20] Wang, Z. , Wu, H., Zhao, G.,Liao, X, Chen, F., Wu, J, Hu, X., "Onedimensional Finite-difference Modeling on Temperature History and Freezing Time of Individual Food", Journal of Food Engineering, Vol. 79 , pp. 502-510, 2007.

[21] Faragó, I. and Palencia, C. "Sharpening The Estimate of The Stability Constant in The Maximum-Norm of The Crank-Nicolson Scheme for The OneDimensional Heat Equation", Applied Numerical Mathematics, Vol. 42, Issues 13, pp 133-140, August 2002,

[22] Welch, G., The Kalman Filter Learning Tool Dynamic and Measurement Models, Chapel Hill, 2003.

[23] Welch, G. and Bishop, G., An Introduction to the Kalman Filter, Chapel Hill, 2001.

[24] Levy, L. J., " Innovation: The Kalman Filter: Navigation's Integration Workhorse", GPS World, Vol. 8, No. 9, 1998.

[25] Khulief, Y. A. and Shabaik, E. H. E., "Laboratory investigation of a multiplemodel state estimation scheme for detection and isolation of leaks in Pipelines", Proceedings of the Institution of Mechanical Engineers, Part I: Journal of Systems and Control Engineering, Vol. 220, No. 1, 2006.

[26] Huange, S.C., Lin, W.W., Tsai, M. T., and Chen, M.H., " Fiber Optic In-Line Distributed Sensor for Detection And Localization of The Pipeline Leaks", Sensors and Actuators, Vol. 135, Issue 2, pp. 570-579, 2007.

[27] Mendoza, M., Carrillo, A., and Marquez, A., "New distributed optical sensor for detection and localization of liquid leaks: Part II. Optimization of the Elastomer Performance", Sensors and Actuators A, Vol. 111, pp. 154:165, 2004

[28] Carrillo, A., Gonzalez, E., Rosas, A., and Marquez, A., "New Distributed Optical Sensor for Detection and Localization of Liquid Leaks: Part I. 
Experimental Studies", Sensors and Actuators A: Physical, Vol. 99, Issue 3, pp. 229-235, 2002.

[29] Kiuchi, T.," A leak localization method of PL by means of fluid transient model", Journal of energy resources technology, Vol. 115, N.3, pp. 162-167, 1993.

[30] Tetzner, R., “Model-based PL Leak Detection and Localization”, 3R International, Pipeline Rehabilitation and Maintenance, Berlin , Vol. 42, pp. 455460, 2003.

Tables:

Table 1: Comparison of Some Leak Detection Methods

\begin{tabular}{|l|c|c|c|c|}
\hline \multicolumn{1}{|c|}{ Method } & Reliability & Sensitivity & Accuracy & Robustness \\
\hline Mass balance & $\mathrm{M}$ & $\mathrm{M}$ & $\mathrm{M}$ & $\mathrm{H}$ \\
\hline Rate of change & $\mathrm{M}$ & $\mathrm{M}$ & $\mathrm{L}$ & $\mathrm{H}$ \\
\hline $\begin{array}{l}\text { Hydraulic } \\
\text { modeling }\end{array}$ & $\mathrm{M}$ & $\mathrm{H}$ & $\mathrm{M}$ & $\mathrm{L}$ \\
\hline $\begin{array}{l}\text { Pressure point } \\
\text { analysis }\end{array}$ & $\mathrm{M}$ & $\mathrm{M}$ & $\mathrm{L}$ & $\mathrm{H}$ \\
\hline $\begin{array}{l}\text { Proposed } \\
\text { method }\end{array}$ & $\mathrm{H}$ & $\mathrm{H}$ & $\mathrm{H}$ & $\mathrm{H}$ \\
\hline
\end{tabular}

Table 2: Average of residuals

\begin{tabular}{|c|c|c|c|c|}
\hline \multirow{2}{*}{$\begin{array}{c}\text { Measurement } \\
\text { station }\end{array}$} & \multicolumn{2}{|c|}{ No leak } & \multicolumn{2}{|c|}{ Single leak } \\
\hline & $\begin{array}{l}\text { Pressure } \\
\text { (bar) }\end{array}$ & $\begin{array}{c}\text { Flow rate } \\
(\mathrm{kg} / \mathrm{s})\end{array}$ & $\begin{array}{c}\text { Pressure } \\
\text { (bar) }\end{array}$ & $\begin{array}{c}\text { Flow rate } \\
(\mathrm{kg} / \mathrm{s})\end{array}$ \\
\hline 1 & $3.790 \times 10^{-4}$ & $6.442 \times 10^{-6}$ & $1.000 \times 10^{-4}$ & 1.885 \\
\hline 2 & $1.077 \times 10^{-4}$ & $1.611 \times 10^{-6}$ & $61.000 \times 10^{-4}$ & 1.244 \\
\hline 3 & $1.244 \times 10^{-4}$ & $9.759 \times 10^{-6}$ & $65.000 \times 10^{-4}$ & 0.000 \\
\hline Average & $2.0370 \times 10^{-4}$ & $5.9373 \times 10^{-6}$ & $42 \times 10^{-4}$ & 1.0429 \\
\hline
\end{tabular}




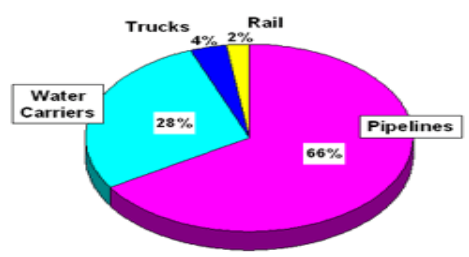

Fig.1. Oil Transportation

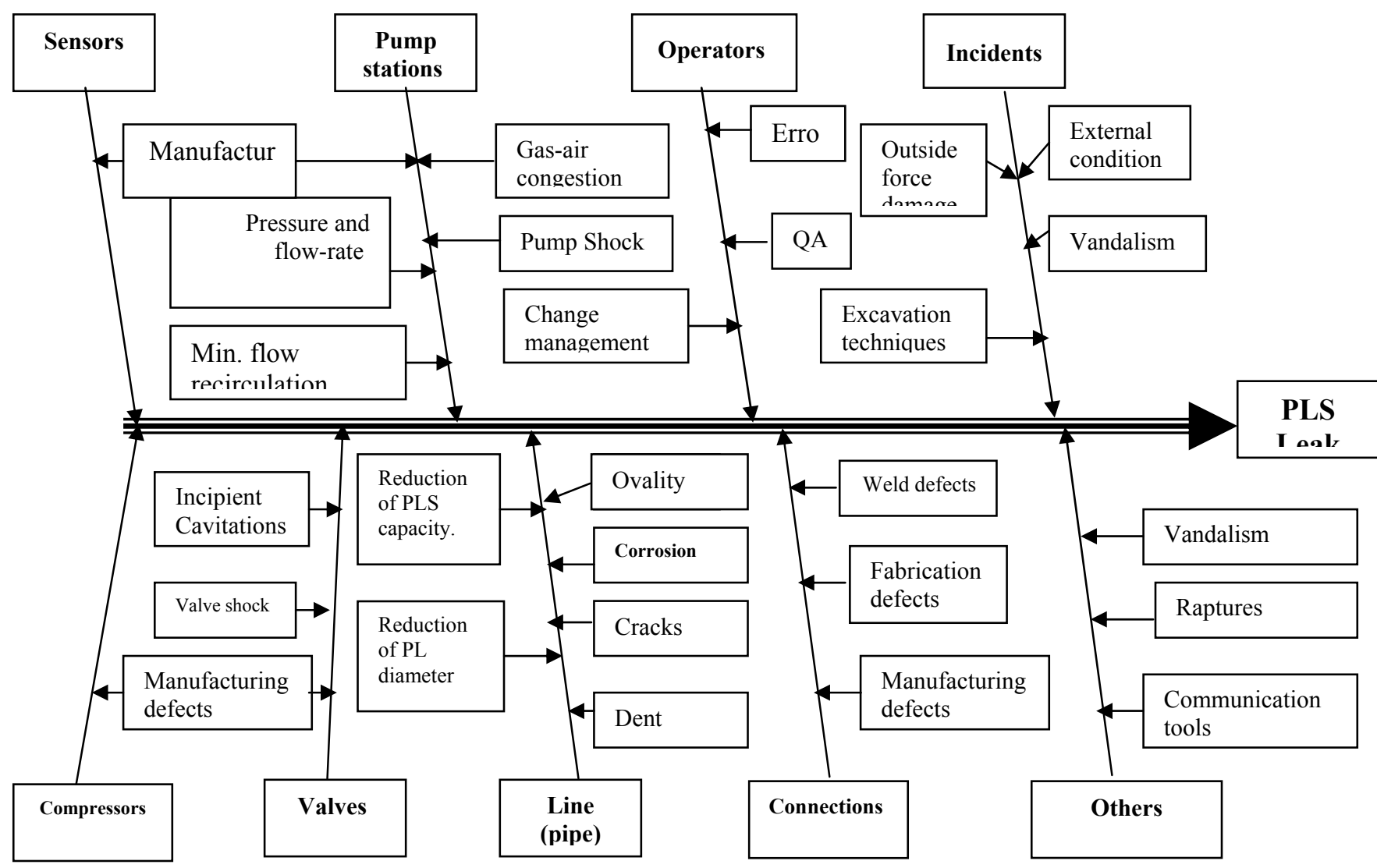

Fig. 2. Detailed Leak Cause-and-Effect diagram for PLS

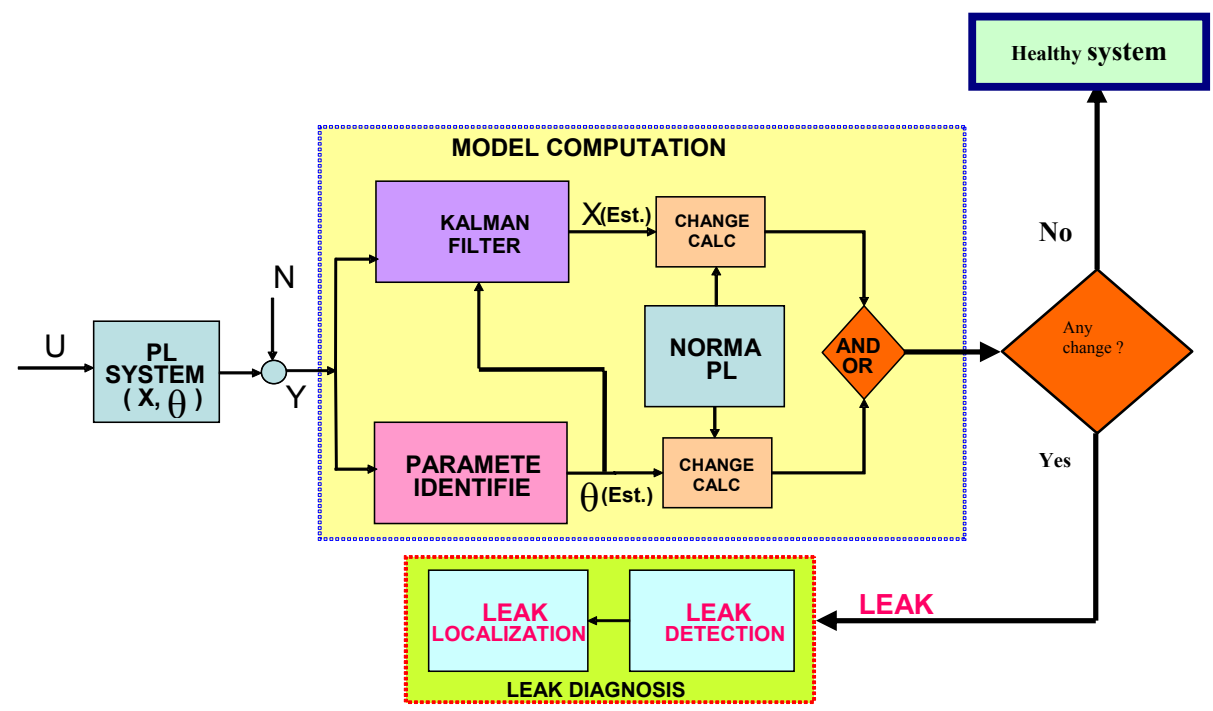

$\mathrm{X}$ is the system state.

$\Theta$ is the system parameter.

$\mathrm{X}_{\text {est }}$ is the estimated state variable

$\Theta_{\text {est }}$ is the estimated pipeline system parameter.

$\mathrm{U}$ is the input to the pipeline system.

$\mathrm{Y}$ is the output of the system. $\mathrm{N}$ is the process noise.

Fig. 3. Flowchart of the generalized leak 
Simulated leak position at midpoint of pipeline $=(\mathrm{N}+1) / 2$

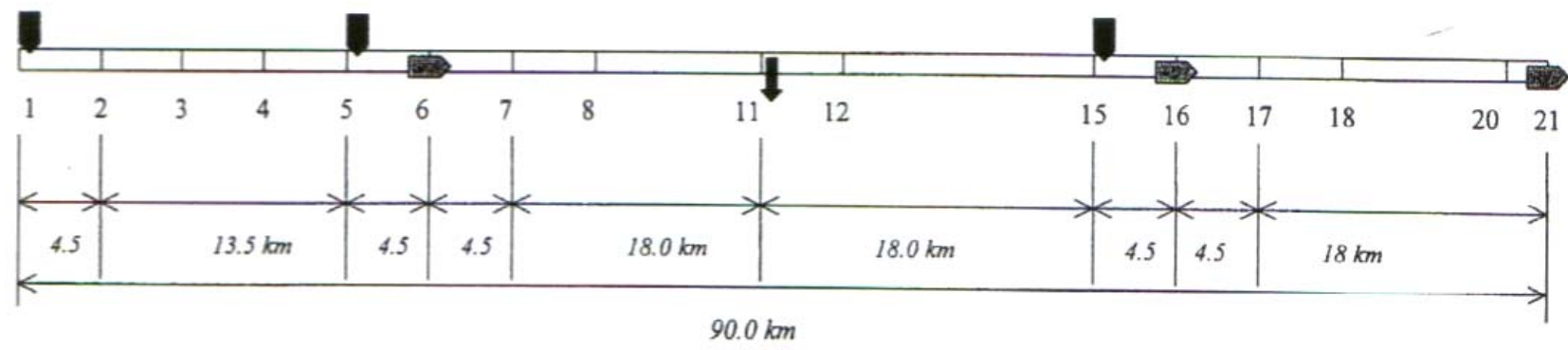

Indicates to pressure transducer

Indicates to flow-rate transmitter

Fig. 4. : Equal segments pipeline with $\mathrm{N}=21$ nodes.

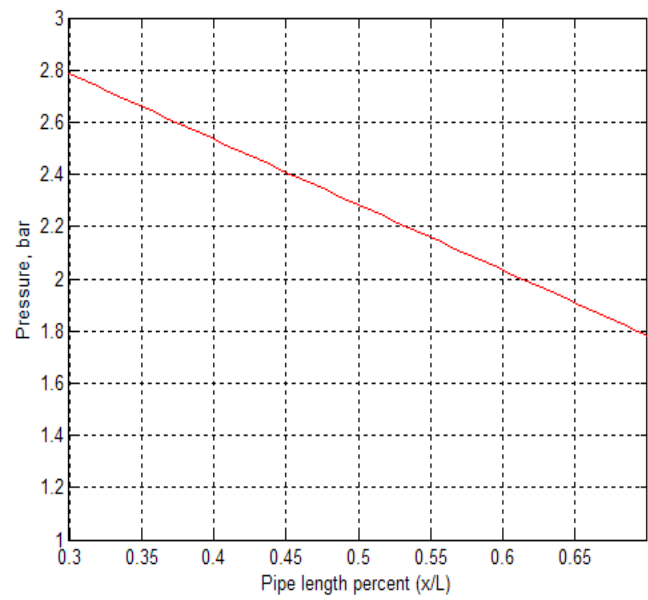

Fig. 5: Pressure profile at final time, no leak case.

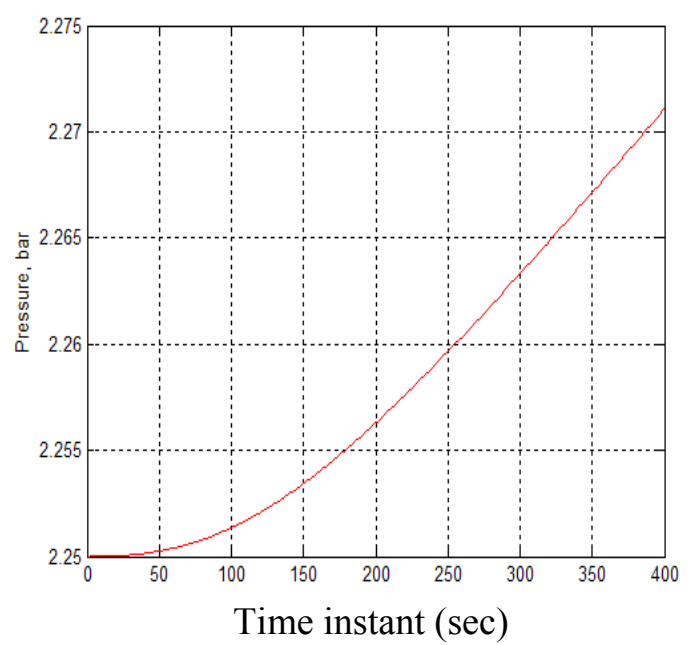

Fig. 7: Pressure Response at middle node; no leak case.

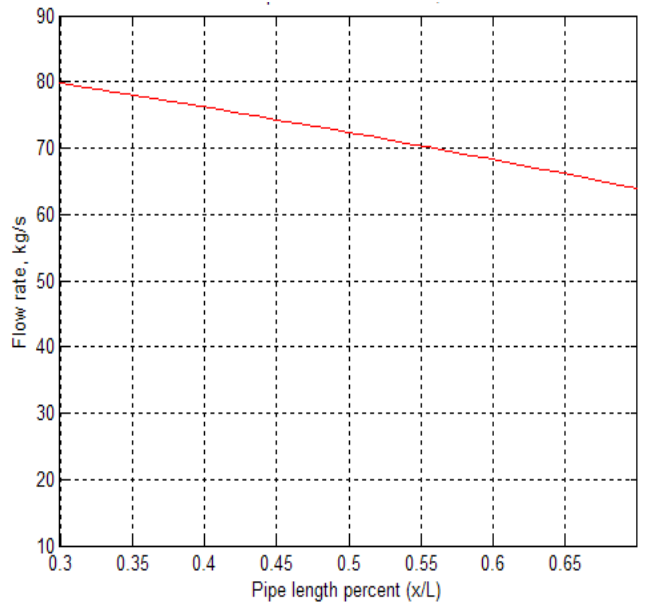

Fig. 6: Flow-rate profile at final time, no leak case.

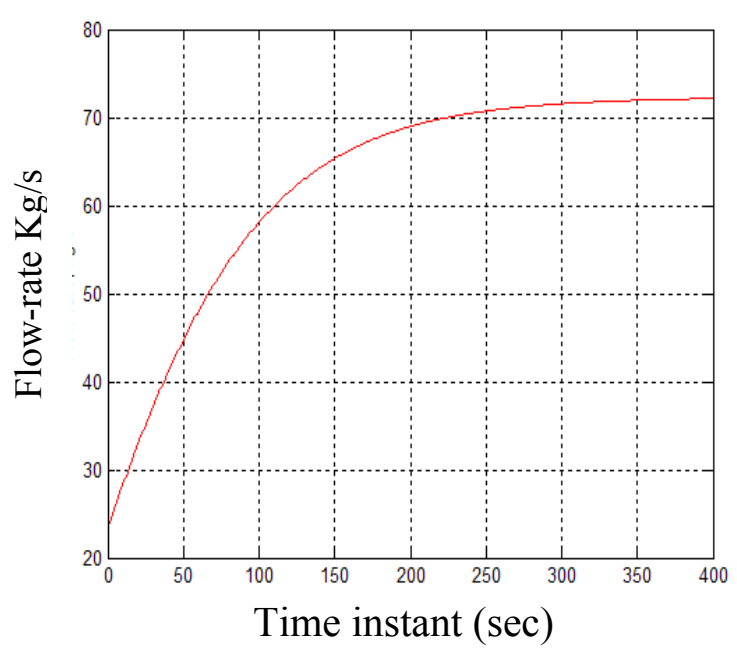

Fig. 8: Flow-rate Response at middle node; no leak case. 


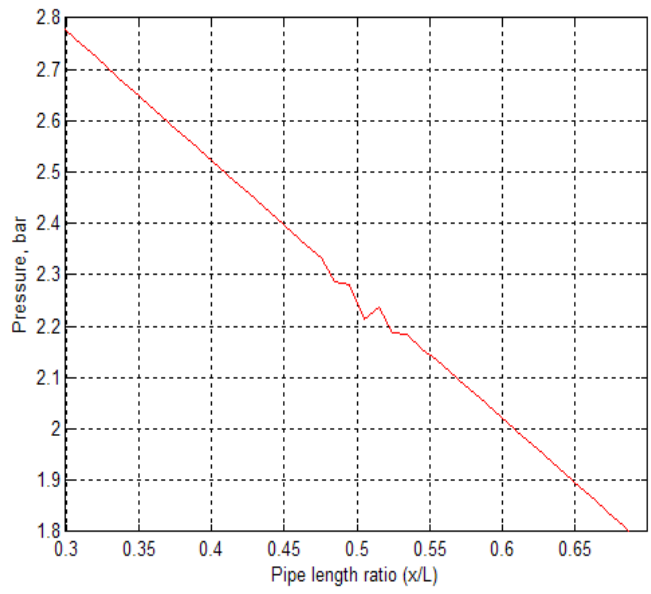

Fig. 9:. Pressure profile before $\&$ after leakage (50 s after onset of leak): single leak case.

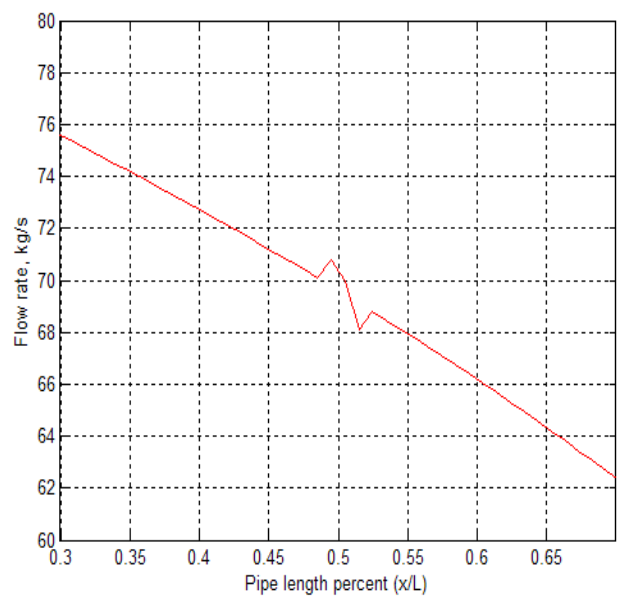

Fig. 11: Flow-rate profile after onset of leak by 10 s; single leak case.

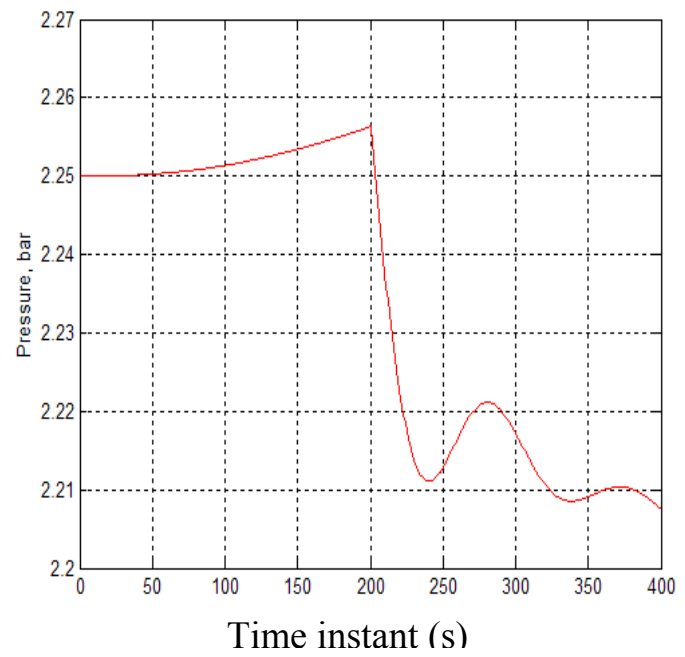

Fig. 13: Pressure response at leak location; single leak case.

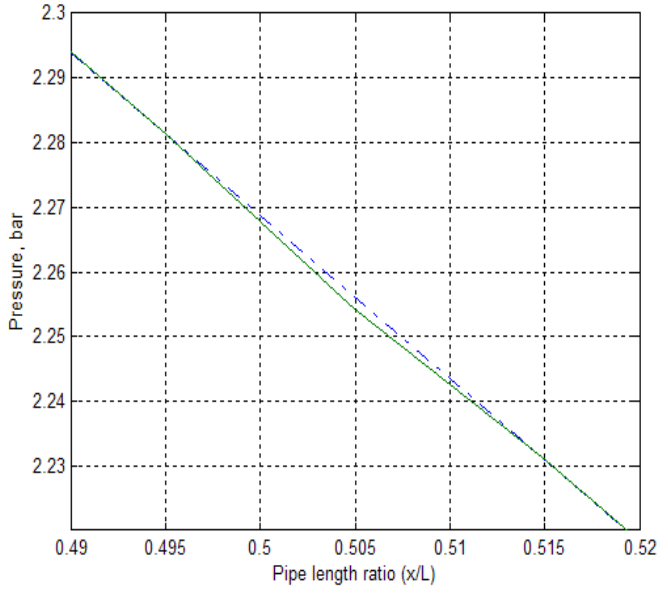

Fig. 10: Pressure profile just before (dashdot) and just after (solid) onset of leak; single leak case.

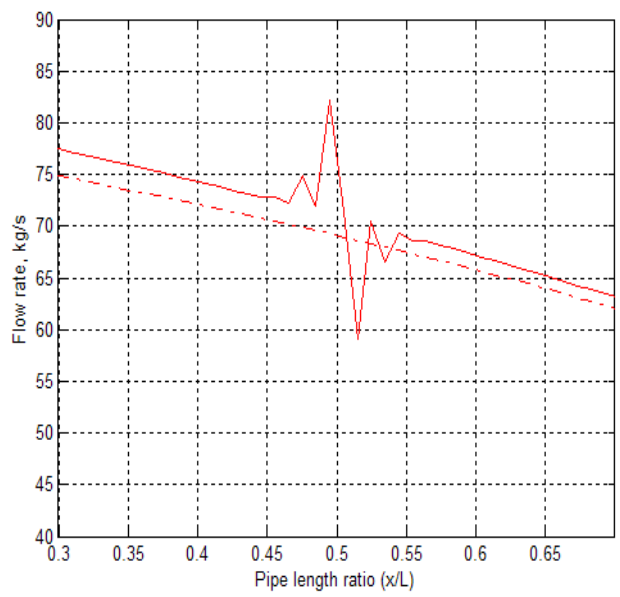

Fig. 12: Flow-rate profile before (dashdot) and 50 s after onset of leak (solid); single leak case.

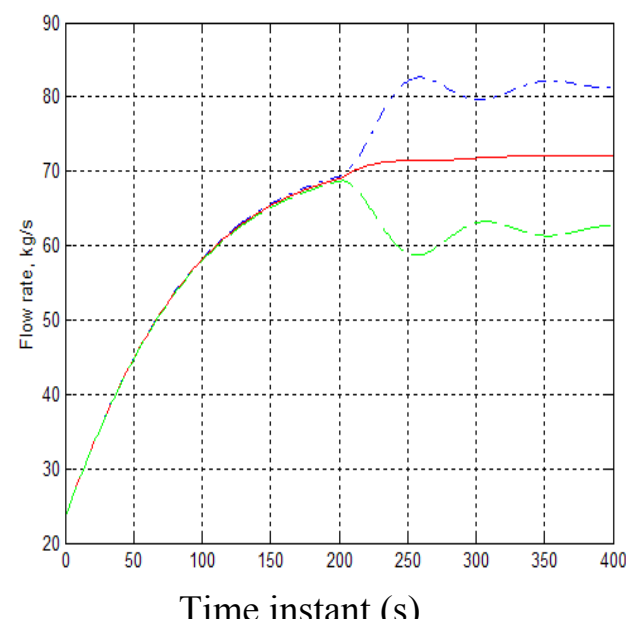

Fig. 14: Flow rate response at upstream (dashdot) and downstream (dash) of leak location; single leak case. 


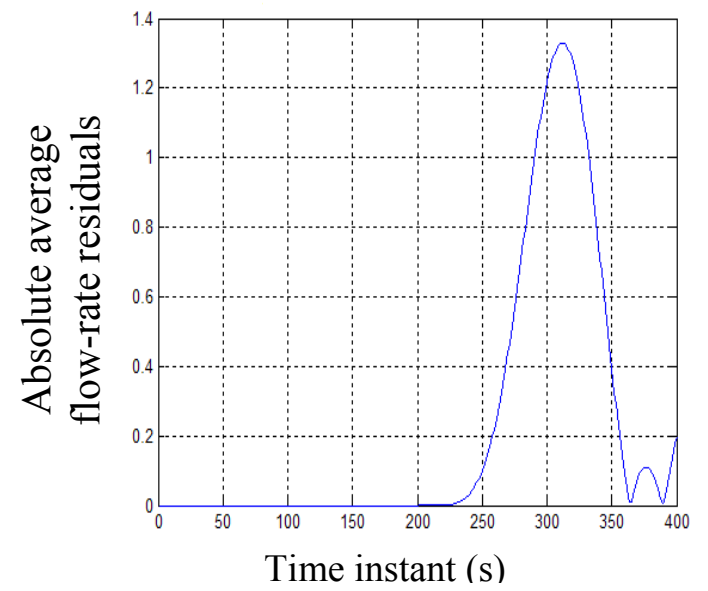

Fig. 15: Absolute average Residuals of Flowrate along Pipeline vs Time

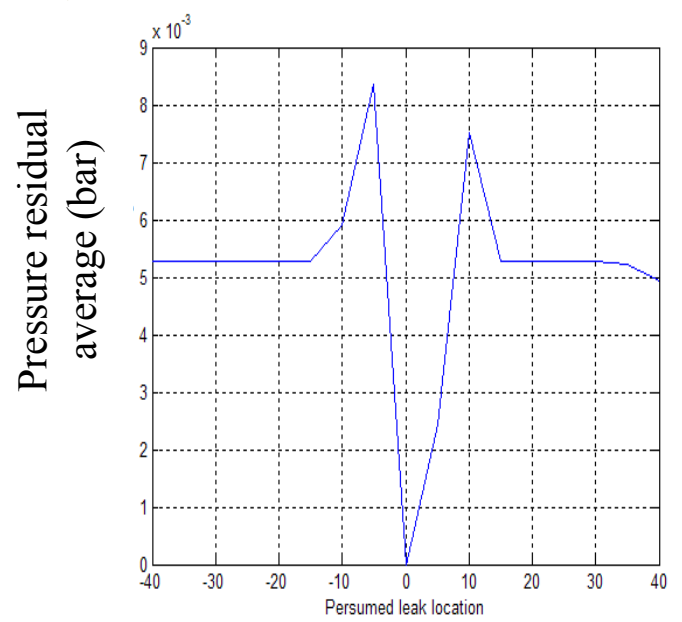

Leak rate $(\mathrm{Kg} / \mathrm{s})$

Fig. 17: Pressure residual average for leak rate $10 \mathrm{~kg} / \mathrm{s}$

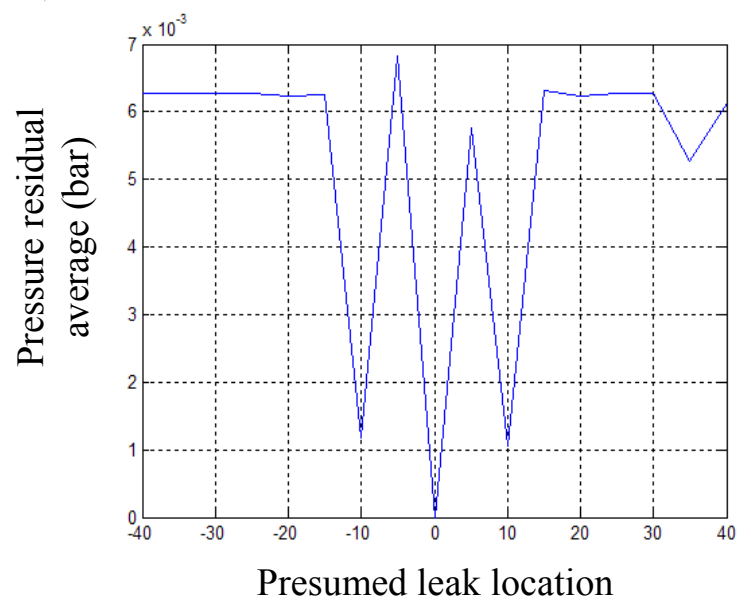

Fig.19: Pressure residual average for leak rate $5 \mathrm{~kg} / \mathrm{s}$

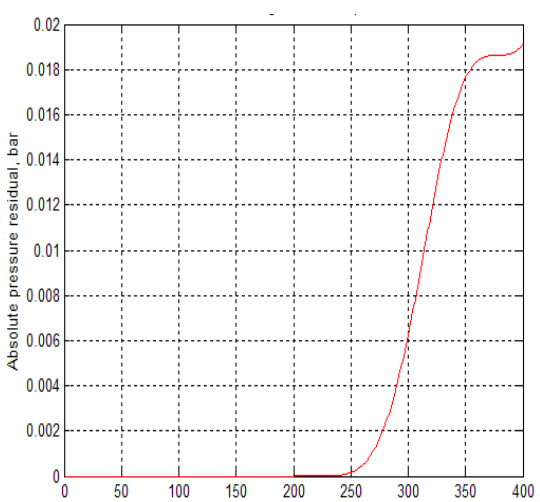

Time instant (s)

Fig. 16: Absolute average Residuals of Pressure along Pipeline Vs Time

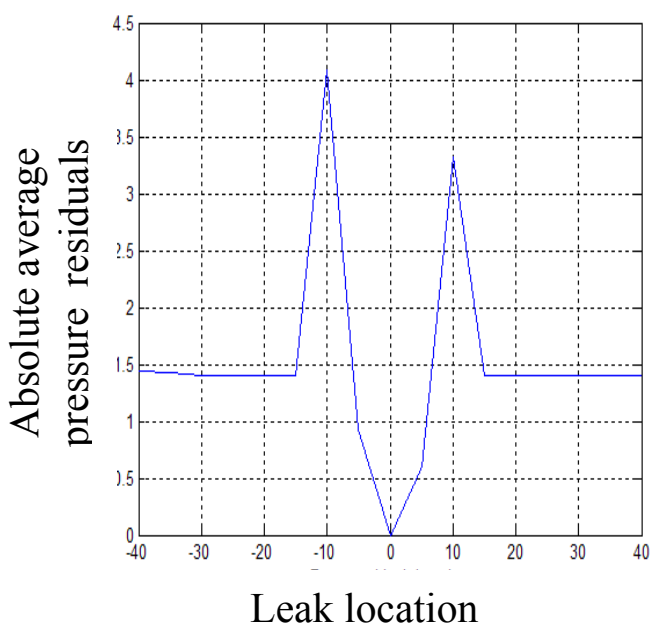

Fig.18: Flow-rate residual average for leak rate $10 \mathrm{~kg} / \mathrm{s}$

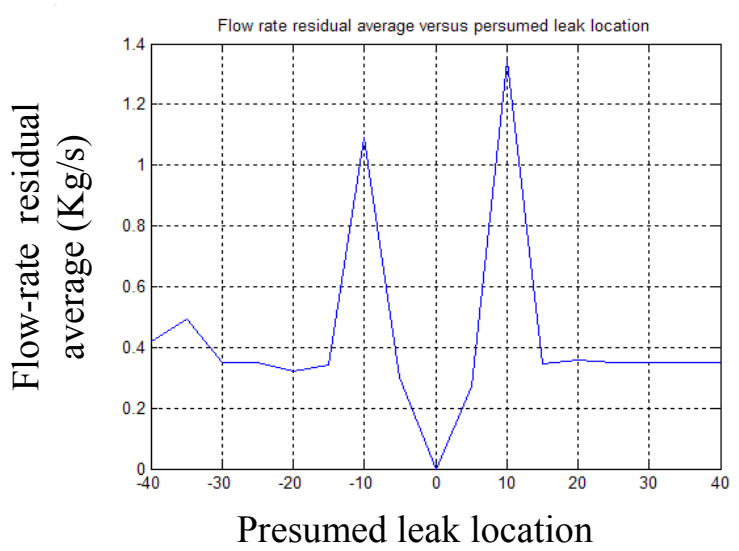

Fig.20 : Flow-rate residual average for leak rate $5 \mathrm{~kg} / \mathrm{s}$ 


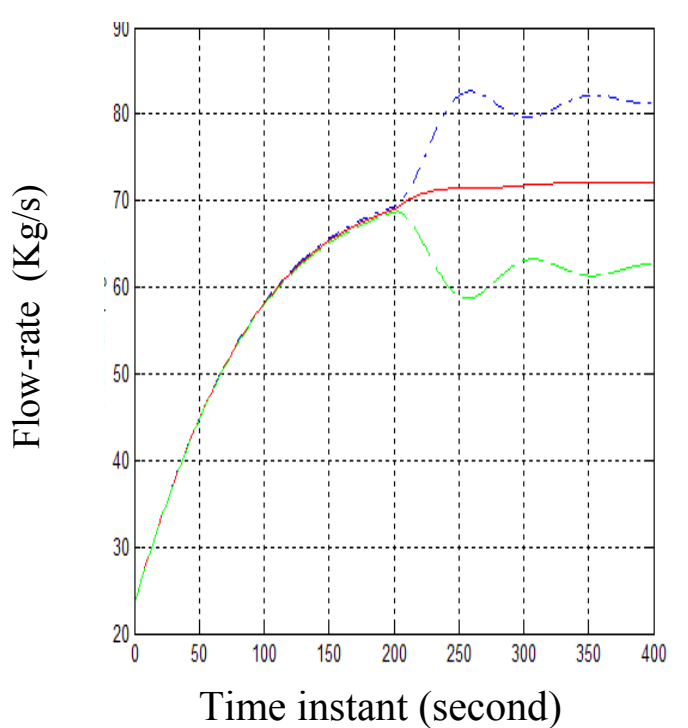

Fig. 21: Flow-rate response for leak and healthy pipelines. Upstream (dashdot), down stream (dash)

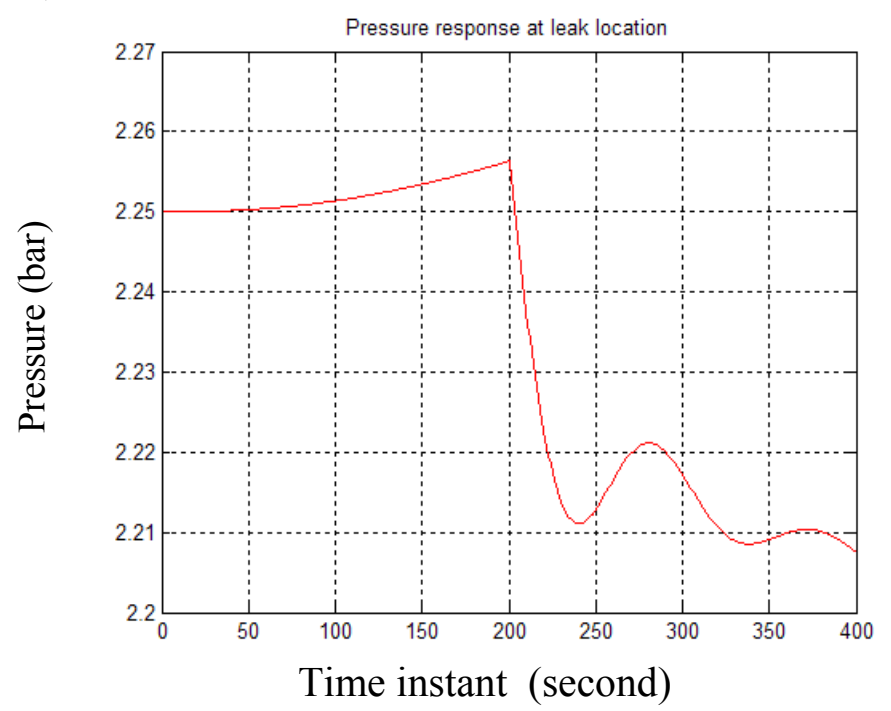

Fig.22: Pressure response for a leaky pipeline measured at the leak location.

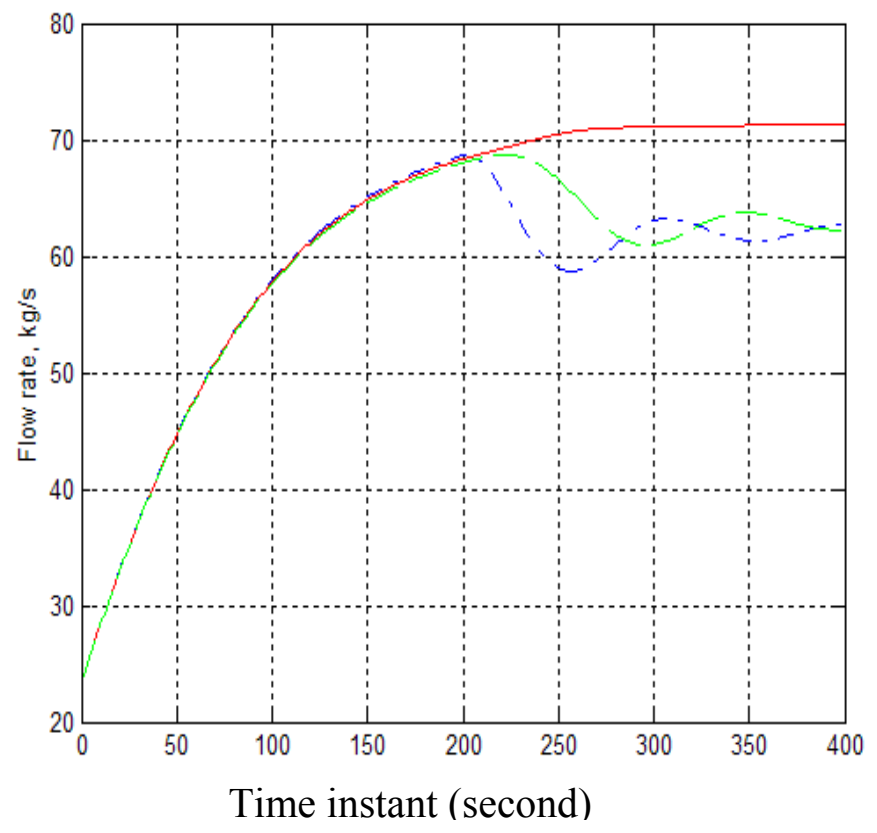

Fig.23: Flow-rate response at two consecutive nodes just downstream the leak 